\title{
ANALYSIS OF INVERTERS AND DC/DC CONVERTERS
}

\author{
R.D. MIDDLEBROOK, Session Chairman
}

Although this is the fifth conference in the series, it is only the third that has included a session on Analys is Techniques. The session was introduced into the program in the belief that this area deserved greater attention and would provide a cornerstone to which application oriented sessions could be referred. In its two previous years, the Analys is session has been well received, and this year four out of the six papers present developments or extensions of work reported in the previous years.

The first paper, by Lee and Wilson, shows how several LC tuned inverters can be considered as examples of a single class reducible to an equivalent circuit containing three series elements, and employs a phase-plane technique to investigate its properties. The second paper, by Wilson, Lee, Burns, and Owen, draws an analogy between this class and another class of saturable reactor inverters previously discussed by the same research group, and shows how the classification procedure suggests identification of four additional inverter classes.

The second paper, by Wester, describes the loop gain analysis and design shaping for a particular dc-to-dc converter. The circuit configuration employs a two-loop feedback topology introduced by Schwarz, the principle of which was reported on by Lalli and Schoenfeld at the 1972 PESC, and also a tapped inductor boost circuit described by Wester at the 1973 PESC and analyzed by a low-frequency averaged model introduced at the 1972 PESC.

The paper by Cape], Ferrante, and Prajoux is also an extension of work previously reported at the 1973 PESC. A z-transform analysis technique is used to examine the stability limits of a two-1oop feedback buck converter, and it is shown that the discontinuous conduction mode of the filter inductor permits higher loop gain to be employed than does the more familiar continuous conduction mode.

The fifth paper, by Walk and Rootenberg, employs control theory concepts whereby the stability of switching-mode regulators can be examined formally by means of a modified Nyquist plot. This approach leads to sufficient but perhaps not necessary conditions for stability, in contrast to the more familiar low-frequency approximation methods which lead usually to necessary but not sufficient conditions for stability.
The paper by Lukens presents an alternative approach to the derivation of the low-frequency averaged model of the three basic switching converter configurations introduced by Wester at the 1972 PESC, and gives experimental confirmation of the resulting describing-function prediction of the buck-boost converter in the presence of an input filter.

Finally, the paper by Schwarz is concerned with the transmission of line disturbances to the output of a feedback switching converter, and with the effect of the loop gain magnitude upon the switching ripple that appears in the output. It is shown that a particular relation between the loop gain and the ratio of the switching frequency to the filter cutoff frequency should be avoided, otherwise the presence of the feedback loop actually increases, rather than decreases, the output switching frequency ripple.

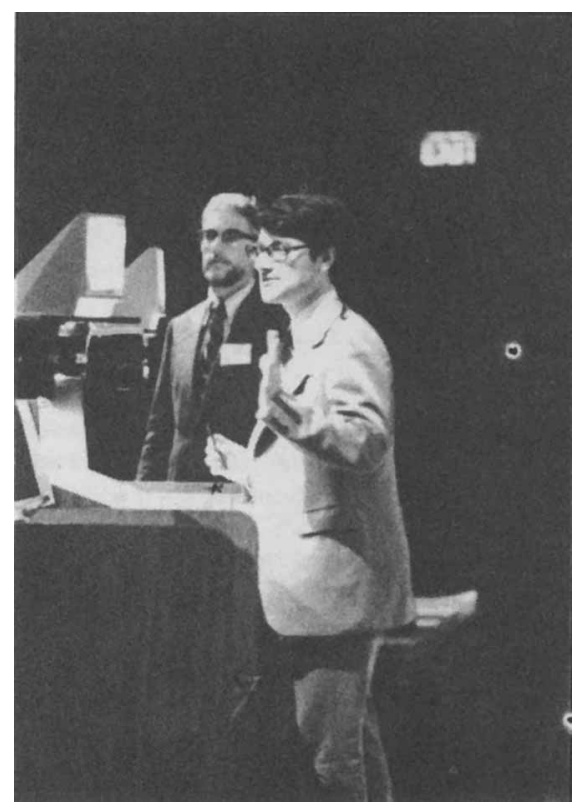

